Carlos Alberto Carrasco* Édgar Demetrio Tovar-García"

Recibido: 17 de julio de 2018 Concepto de evaluación: 13 de febrero de 2019 Aprobado: 12 de diciembre de 2019

Artículo de investigación (c) 2019 Universidad Católica de Colombia. Facultad de Ciencias Económicas y Administrativas. Todos los derechos reservados

* Doctor en Integración Económica Vasco; docente-investigador de Economía en la Universidad de Monterrey (UDEM, México) y miembro del Sistema Nacional de Investigadores (SNI-1 CONACyT). Dirección de correspondencia: Av. Ignacio Morones Prieto 4500 Pte., 66238, San Pedro Garza

García, N.L., México. Correo electrónico: carlos.carrasco@udem.edu

(D) https://orcid.org/0000-0002-5439-4960

** Doctor en Economía; docenteinvestigador de Economía en la Universidad

Panamericana, Escuela de Ciencias Económicas y Empresariales; miembro del Sistema Nacional de Investigadores (SNI-2 CONACyT). Dirección de correspondencia: Álvaro del Portillo 49, Zapopan, Jalisco, 45010, México. Correo electrónico: dtovar@up.edu.mx

(D) https://orcid.org/0000-0002-8826-0004
Finanz. polit. econ., ISSN: 2248-6046, Vol. 11, N. ${ }^{\circ} 2$, julio-diciembre, 2019, pp. 259-276

http://doi.org/10.14718/revfinanzpolitecon.2019.11.2.3

\section{Determinantes del balance comercial bilateral de México: ingreso, tipo de cambio y composición de las exportaciones}

\section{RESUMEN}

En este artículo se utilizan modelos dinámicos con datos de panel para estudiar los determinantes del balance comercial bilateral de México con 39 socios comerciales en el periodo 1990-2016. La literatura señala al ingreso y al tipo de cambio real como las variables independientes clave. Asimismo, se incluye la composición de las exportaciones como variable explicativa (algo ignorado previamente). Los resultados difieren de otras investigaciones: no se encuentra evidencia robusta sobre el efecto del ingreso, hay evidencia de la importancia del tipo de cambio real y se encuentra un nexo positivo, aunque débil, con las exportaciones consistentes de bienes de alta tecnología, lo cual explica parcialmente el persistente déficit comercial de México.

Palabras clave: balance comercial bilateral, composición de las exportaciones, industrias de alta intensidad tecnológica, México.

JEL Classification: F14, F41, O24

Cómo citar este artículo / To reference this article / Para citar este artigo:

Carrasco, C. A. \& Tovar-García, É., D. (2019). Determinantes del balance comercial bilateral de México: ingreso, tipo de cambio y composición de las exportaciones. Revista Finanzas y Política Económica, 11(2), 259-276. doi: http://dx.doi.org/10.14718/ revfinanzpolitecon.2019.11.2.3

\section{Determinants of Mexico's Bilateral Trade Balance: Income, Exchange Rate, and Export Composition}

\section{ABSTRACT}

Using dynamic panel data models, this paper studies the determinants of Mexico's bilateral trade balance with 39 trade partners from 1990 to 2016. The literature points to income and real exchange rate as key independent 
variables. This study includes export composition as an explanatory variable (a previously neglected aspect). Results differ from other researches: there is no robust evidence on the effect of income; there is evidence of the association between bilateral balance and real exchange rate; and there is a weak positive link with exports consisting of high-tech goods, partially explaining Mexico's persistent trade deficit.

Keywords: Bilateral trade balance, export composition, high-tech industries, Mexico.

\section{Determinantes da balança comercial bilateral mexicana: renda, taxa de câmbio e composição das exportações}

\section{RESUMO}

Neste artigo, são utilizados modelos dinâmicos de dados em painel a fim de estudar os determinantes da balança comercial bilateral mexicana com 39 parceiros comerciais de 1990 a 2016. A literatura indica que a renda e a taxa de câmbio real são as principais variáveis independentes. Aqui, a composição da exportação é incluída como variável explicativa, o que não era considerado antes. Os resultados diferem de outras pesquisas: não há evidências sólidas sobre o efeito da renda; há evidências da importância da taxa de câmbio real e verifica-se uma associação positiva, embora fraca, com as exportações de bens de alta tecnologia, o que explica parcialmente o persistente déficit comercial do México.

Palavras-chave: balança comercial bilateral, composição das exportações, indústrias de alta tecnologia, México. 


\section{INTRODUCCIÓN}

La vulnerabilidad asociada a la presencia y persistencia de desequilibrios externos es especialmente relevante en el caso de los países en desarrollo (Calderón, Chong y Loayza, 2002; Dooley, 2000; Mishkin, 1996). No obstante, las preocupaciones por la sostenibilidad de los grandes desequilibrios en la cuenta corriente han tenido lugar en los grandes bloques económicos, incluyendo a países desarrollados (Krugman y Baldwin, 1987; Brissimis et al., 2013; Carrasco y Serrano, 2015). México no ha sido la excepción, pues presenta un déficit persistente en la cuenta corriente y, a la vez, un superávit con respecto a Estados Unidos (Galindo y Guerrero, 1997; Varela, 1999; Garcés, 2008; Baltazar y Ramos, 2014; Arriaga y Landa, 2016; Lagunes y Pérez, 2016; Márquez, 2018).

El análisis de los determinantes de los desbalances en el sector externo permite la actuación de la política económica en determinados aspectos necesarios de corregir. En México, desde los ochenta se ha llevado a cabo un profundo proceso de reforma que ha incluido la apertura comercial y financiera. Así, existen dos momentos clave en el proceso de reforma del sector externo: la entrada al Acuerdo General sobre Aranceles Aduaneros y Comercio (apertura unilateral en 1986) y la entrada en vigor del Tratado de Libre Comercio de América del Norte (TLCAN, con Estados Unidos y Canadá en 1994). En esta línea de apertura económica, a mayo del 2015 México contaba con 12 tratados de libre comercio con 46 países (Secretaría de Economía, 2015).

Desde los ochenta, el comercio exterior de México ha mantenido una tendencia creciente. Con datos de los Indicadores Mundiales de Desarrollo del Banco Mundial, la apertura comercial en 1980 era de 23,7\%, mientras que al inicio del proceso de apertura comercial unilateral (1986) fue de 30,8\%. El cambio más importante llegó con la entrada en vigor del TLCAN: en 1994 la apertura comercial fue de 29,3\%, en 1995 fue de 46,1\% y para 2016 había llegado al 78,1\%. Cabe notar que el crecimiento significativo de las exportaciones fue acompañado por un incremento de similar magnitud en las importaciones.
No obstante, dejando de lado la relación con Estados Unidos, el balance externo de la economía mexicana ha tenido un sesgo deficitario. En contabilidad nacional, el balance externo - medido como el saldo en la cuenta corriente- expresa decisiones de ahorro-inversión a partir de la diferencia entre el ahorro nacional y la inversión. En el largo plazo, la presencia de déficits persistentes en cuenta corriente se verá reflejada en un incremento de la deuda y, potencialmente, en la vulnerabilidad de la economía ante perturbaciones externas. Adicionalmente, la mayor parte de las operaciones dentro de la cuenta corriente (sumando créditos y débitos) se ha dado en la cuenta de bienes. Con datos de la Organización para la Cooperación y el Desarrollo Económicos (OCDE), la suma de créditos y débitos del comercio de bienes alcanzó 70,6\% del PIB en el 2016, mientras que la suma total de débitos y créditos de todos los componentes de la cuenta corriente llegó al 82,7\% del PIB.

Así, el objetivo de la presente investigación es analizar los determinantes del balance bilateral de bienes en México. Por su naturaleza, los determinantes del balance externo general y del balance bilateral de un país difieren significativamente (Khan y Hossain, 2012); el primero incluye factores agregados, mientras que el segundo examina los factores relativos que influyen en la relación bilateral. Así, en los análisis empíricos del comercio bilateral se han incluido como determinantes al tipo de cambio real bilateral, las diferencias en el nivel de ingresos, la evolución de los costos de transporte, los flujos de inversión extranjera y los costos laborales (Çelik y Kaya, 2010; Khan y Hossain, 2010, 2012; Gu, Zhou y Beg, 2014; Bineau, 2016).

Por otra parte, diversos estudios han señalado la relevancia de la composición de las exportaciones para el crecimiento económico, la productividad y el desempeño de las exportaciones (Ghatak, Milner y Utkulu, 1997; Aditya y Acharyya, 2013; Wierts, Van Kerkhoff y De Haan, 2014). No obstante, la composición de las exportaciones - y con ello la especialización de la industria exportadora- no se ha incluido en el análisis del balance comercial bilateral de México, aunque existe evidencia de 
su importancia para otros países (Tovar-García y Carrasco, 2019).

La relación entre el saldo en el balance comercial bilateral y la composición de las exportaciones se asocia a la elasticidad de precio e ingreso de los bienes. En este sentido, cuando una proporción alta de las exportaciones se compone de bienes con una elasticidad de precio e ingreso relativamente baja, la capacidad explicativa de factores asociados al precio (tipo de cambio real) y al ingreso (nivel relativo de ingreso) pierde significancia, dando paso a la composición de las exportaciones como determinante fundamental. Dos grupos de bienes tendrían potencialmente una elasticidad baja: el petróleo y el gas natural - como insumos clave dentro del sistema productivo internacional que no cuentan con bienes sustitutos a precios competitivos-, y los bienes de alta intensidad tecnológica - cercanos a la frontera tecnológica y con fuentes relativamente limitadas de competencia- (Cooper, 2003; Krichene, 2002).

Este trabajo es pionero en el análisis de las relaciones anteriores para el caso de México. Dado que la participación de las exportaciones de petróleo ha disminuido, mientras que la de los bienes manufactureros ha ido en aumento, el estudio se centra en las exportaciones de industrias con alta intensidad tecnológica y su influencia en la determinación del balance bilateral de la economía mexicana. Los estudios empíricos previos han incluido el nivel de ingreso relativo y el tipo de cambio real bilateral como determinantes claves; aquí se verifica su relevancia para el caso mexicano, superando las limitaciones de los estudios anteriores centrados en el nivel de ingreso de Estados Unidos y en el tipo de cambio peso-dólar.

Además de esta introducción, el texto se compone de cuatro secciones más. La siguiente hace una revisión de la literatura y presenta algunas gráficas para describir la conexión entre el desempeño del balance externo y la composición de las exportaciones. Una sección posterior expone y describe las principales variables, así como la estrategia metodológica a seguir, implementando distintos modelos de panel dinámico que proveen robustez y consistencia a los hallazgos. Luego se presentan los principales resultados, y finalmente se exponen las conclusiones pertinentes.

\section{LA CONEXIÓN ENTRE LA COMPOSICIÓN DE LAS EXPORTACIONES Y EL BALANCE BILATERAL EXTERNO EN MÉXICO}

La literatura económica ha analizado el papel de la composición de las exportaciones en el desempeño del sector exportador, la productividad y el crecimiento económico (Ghatak, Milner y Utkulu, 1997; Aditya y Acharyya, 2013; Wierts, Van Kerkhoff y De Haan, 2014), pero no ha profundizado en su papel como determinante del balance bilateral externo; sin embargo, recientemente se ha señalado su importancia tanto como determinantes de la demanda por exportaciones (Tovar, 2018; Wierts, Van Kerkhoff y De Haan, 2014) como en términos del balance bilateral (Tovar y Carrasco, 2019). La conexión entre estas variables se asocia a una transición de la exportación de bienes primarios a manufacturados, con externalidades positivas para el resto de la economía (Fosu, 1990; Ghatak, Milner y Utkulu, 1997; Herzer, Nowak y Siliverstovs, 2006).

Los incrementos en la productividad y los efectos en el crecimiento generados por las exportaciones se explican por tres razones fundamentales (Herzer, Nowak y Siliverstovs, 2006): a) las exportaciones promueven la especialización en sectores con ventaja comparativa, lo que influye en la relocalización de recursos hacia áreas más productivas; b) el acceso a un mercado más grande permite la presencia de economías de escala; c) las industrias exportadoras pueden generar externalidades positivas para el resto de la economía.

Por otro lado, solo recientemente se ha subrayado la relación entre el balance externo y las exportaciones consistentes de bienes con un alto valor añadido ${ }^{1}$, lo cual ha servido para explicar los

\footnotetext{
1 Un problema fundamental es que se pueden contabilizar las exportaciones brutas como de industrias de valor agregado alto, aunque solo una pequeña parte de ese valor se haya creado en el país exportador (Johnson, 2014). Por tanto, parte de lo atribuido como exportaciones de industrias con
} 
desequilibrios externos en la eurozona (Wierts, Van Kerkhoff y De Haan, 2014; Carrasco y Peinado, 2015; Carrasco y Hernandez, 2017). Así, los países con una alta proporción de exportaciones de bienes de alto valor añadido - como los bienes de alta tecnología - presentan una estructura económica que les permite adaptarse al entorno cambiante de la economía mundial, absorber a personal altamente cualificado y producir bienes que se diferencian en términos de calidad, variedad y exclusividad, lo que a su vez reduce la vulnerabilidad de sus exportaciones ante cambios en los precios y el ingreso.

La literatura empírica ha explorado la relación entre la composición de las exportaciones y el desempeño de las economías. Por una parte, se ha puesto de manifiesto que tanto la diversificación de las exportaciones como su composición son factores que influyen en el crecimiento económico (Ghatak, Milner y Utkulu, 1997; Aditya y Acharyya, 2013), aunque los efectos positivos de las exportaciones en la productividad y el crecimiento son limitados cuando se trata de exportaciones de bienes primarios, y mayores cuando se exportan manufacturas (Fosu, 1990; Ghatak, Milner y Utkulu, 1997; Herzer, Nowak y Siliverstovs, 2006).

En este contexto, los efectos positivos sobre la producción se dan especialmente en los países con una proporción de exportaciones de manufacturas mayor que el promedio mundial (Aditya y Acharyya, 2013), mientras que la relación positiva entre exportaciones de manufacturas y crecimiento económico se cumple cuando se ha superado cierto umbral de desarrollo, dada la necesidad de contar con un nivel mínimo de capacitación técnica para poder obtener beneficios de la producción de manufacturas (Sheridan, 2014). Así, el crecimiento en las exportaciones de industrias con alta intensidad tecnológica - que requieren mano de obra cualificada- hace que se mejore el nivel educativo medio (Blanchard y Olney, 2017). Entre tanto, las empresas que invierten

alta intensidad tecnológica se explicaría por la fragmentación de las cadenas de valor en el ámbito internacional, sobreestimando las estadísticas de comercialización de estas industrias (Srholec, 2007). más en investigación y desarrollo (I+D) tienden a exportar más, lo que en conjunto tiene efectos positivos en la productividad (Bravo, Benavente y González, 2014; Wierts, Van Kerkhoff y De Haan, 2014).

Sin embargo, los efectos de la composición de las exportaciones sobre el balance bilateral externo no se han examinado a profundidad. Los estudios del balance bilateral externo se enfocan en el papel de factores relativos como el tipo de cambio real bilateral, el nivel relativo de ingreso, los costos de transporte, los flujos de inversión extranjera directa y la evolución de los costos laborales (Bahmani y Saha, 2017; Bahmani y Wang, 2006; Bahmani y Harvey, 2018; Bahmani, Hegerty y Xu, 2013; Bineau, 2016; Çelik y Kaya, 2010; Gu et al., 2014; Khan y Hossain, 2012; Zakir e Ismail, 2010). No obstante, cuando el balance posee una proporción alta de bienes con una elasticidad baja (p. e., petróleo o bienes con intensidad tecnológica alta) es posible que la composición de las exportaciones (o de las importaciones) se torne relevante y el papel del ingreso relativo y los precios pierda poder explicativo, como se ha señalado en el caso de Rusia (Tovar y Carrasco, 2019).

Por lo anterior, el presente análisis se enfoca en los determinantes del balance de bienes y, particularmente, en el papel de la composición de las exportaciones. Para el caso de México, no existen trabajos que evalúen el papel de la composición de las exportaciones en el balance bilateral de bienes. Existen pruebas previas enfocadas en el comercio con Estados Unidos y con estimaciones de la condición Marshall-Lerner (Galindo y Guerrero, 1997; Varela, 1999; Garcés, 2008; Baltazar y Ramos, 2014; Arriaga y Landa, 2016; Lagunes y Pérez, 2016; Márquez, 2018). En esta misma línea, Bahmani, Harvey y Hegerty (2018) se enfocan en el análisis de los determinantes del balance comercial a nivel industrial, utilizando a los Estados Unidos como país de referencia. Si bien es cierto que actualmente el $81 \%$ de las exportaciones mexicanas y el $47 \%$ de las importaciones ocurren con Estados Unidos, al negar la relevancia del resto de los socios comerciales se generan sesgos importantes para explicar el balance comercial. 
¿Por qué enfocarnos en el balance de bienes? Porque la mayoría de las transacciones registradas en la cuenta corriente (más de $80 \%$ ) se da en el comercio de bienes (figura 1). Con datos de la OCDE, la suma de créditos y débitos del comercio de bienes de la economía mexicana pasó de 52\% (\%PIB) a 70,6\% entre el 2006 y el 2016, lo cual que equivale a $84 \%$ y $85 \%$ de la suma de créditos y débitos de la cuenta corriente. Por su parte, en ese mismo periodo el comercio de servicios (créditos más débitos) rondó entre 4,1\% y 5,4\% (\%PIB), mientras que las subcuentas de ingresos primarios e ingresos secundarios se encontraron entre 3,4\% y $4,1 \%$, y entre $2,7 \%$ y $2,6 \%$, respectivamente. En términos netos (créditos menos débitos), con la excepción del balance de ingreso secundario, guiado por el desempeño de las remesas, las subcuentas de la cuenta corriente han presentado un sesgo persistentemente deficitario.

¿Por qué enfocarnos en las exportaciones de industrias intensivas en tecnología como determinantes del balance bilateral de bienes? En México, la apertura comercial implicó un cambio en la estructura de las exportaciones, disminuyendo la proporción de los bienes de extracción (petróleo y gas natural) e incrementando la proporción de bienes manufacturados. Como lo muestra la figura 2 (parte a), en 1990 las exportaciones de bienes de extracción representaban el 33,5\% de las exportaciones de bienes, mientras que en el 2016 solo

Componentes y balances de la cuenta corriente de México
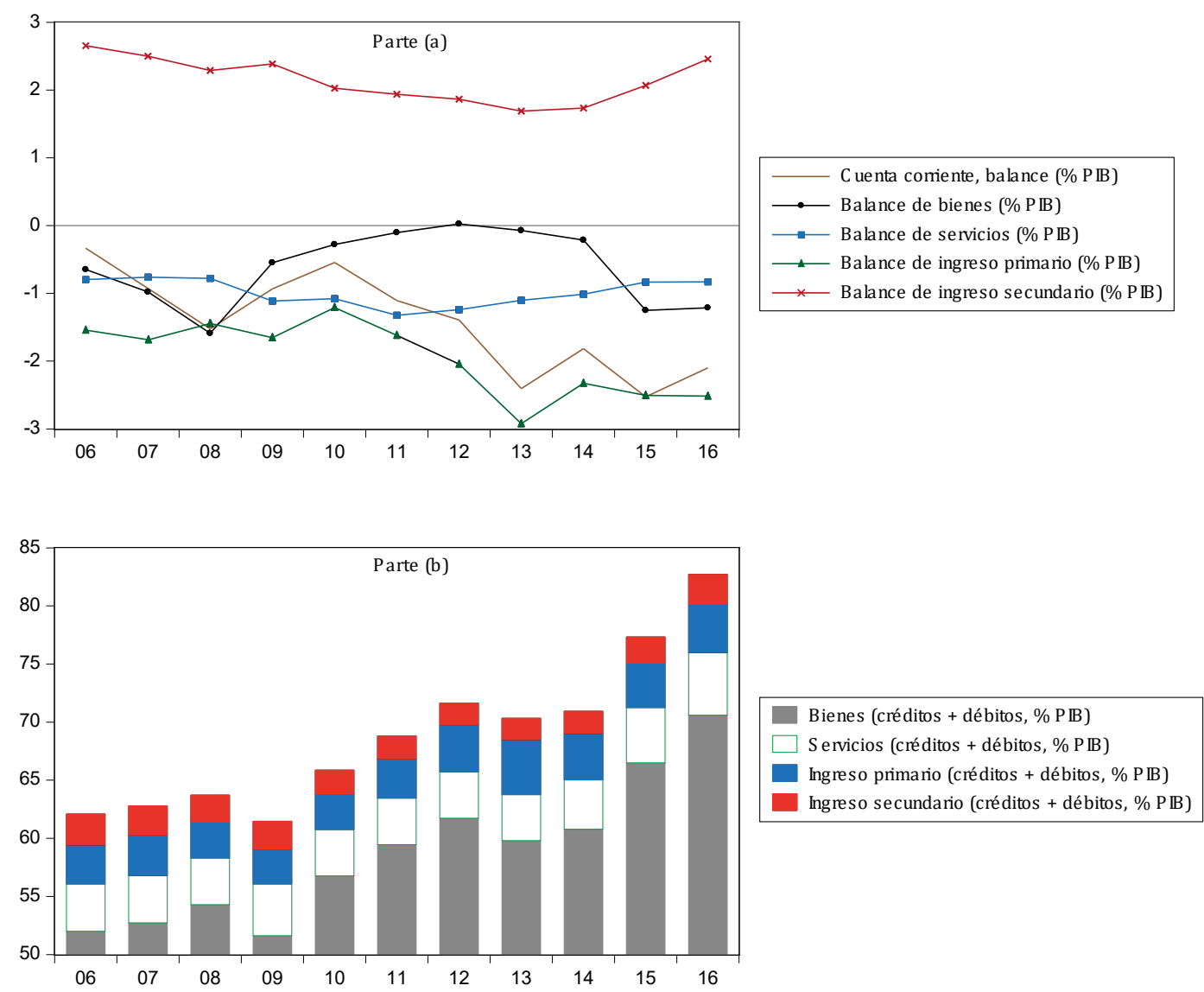

Fuente: elaboración propia a partir de datos de la OCDE, utilizando el programa Eviews. 
alcanzaban la cifra de $4,1 \%$. Por su parte, las exportaciones de bienes manufacturados representaban el $54,7 \%$ de las exportaciones de bienes en 1990 y se incrementaron hasta $89,1 \%$ en el 2016. Una parte importante de este cambio vino del impulso a las manufacturas a partir de la apertura comercial, mientras que otra parte se explica por la caída en el precio del petróleo a mediados de los años ochenta (Moreno y Ros, 2010).

Adicionalmente, se ha observado un cambio en la composición de las exportaciones de manufactura en términos de intensidad tecnológica. La figura 2 (parte b) muestra la composición de las exportaciones de manufacturas en términos de la intensidad tecnológica de las industrias exportadoras. La entrada al TLCAN se reflejó en un incremento en las exportaciones de industrias con alta intensidad tecnológica, que mantuvieron posteriormente un nivel relativamente estable. Más aún, el mayor cambio se dio en las industrias con intensidad tecnológica media-alta, que tienen una participación dominante.

La figura 3 muestra la relación entre el balance externo total de bienes (medido como el cociente entre exportaciones e importaciones de bienes) y la proporción de las exportaciones por distintos niveles de intensidad tecnológica en las exportaciones totales de bienes. Si bien a nivel agregado la relación no es clara para el caso de México, es posible esperar que al analizar los datos bilaterales los resultados

Figura 2.

Composición de las exportaciones de bienes
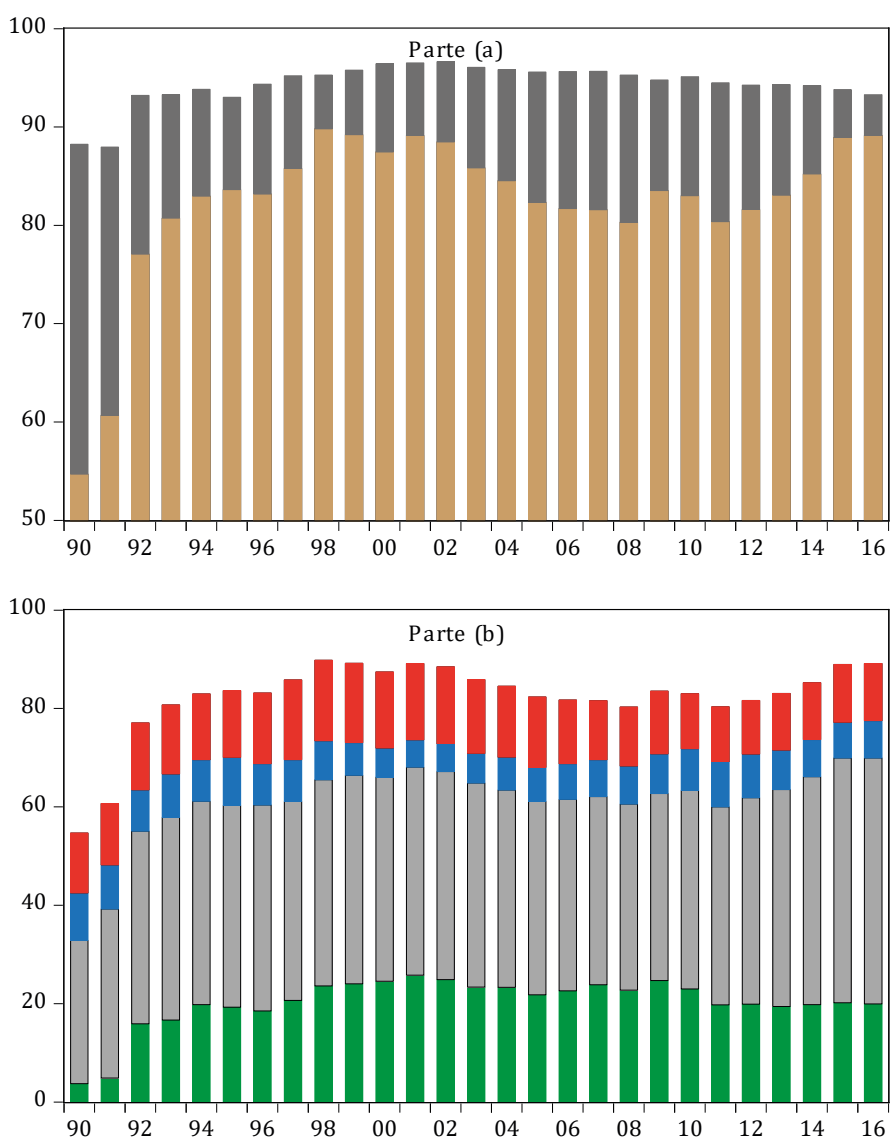

Exportaciones de manufactura (\% exportaciones de bienes) Exportaciones de extracción (petróleo crudo y gas natural, $\%$ exportaciones de bienes

Fuente: elaboración propia a partir de la base de datos STAN de la OCDE, utilizando el programa Eviews. 
Figura 3.

Balance externo de bienes y exportaciones por intensidad tecnológica
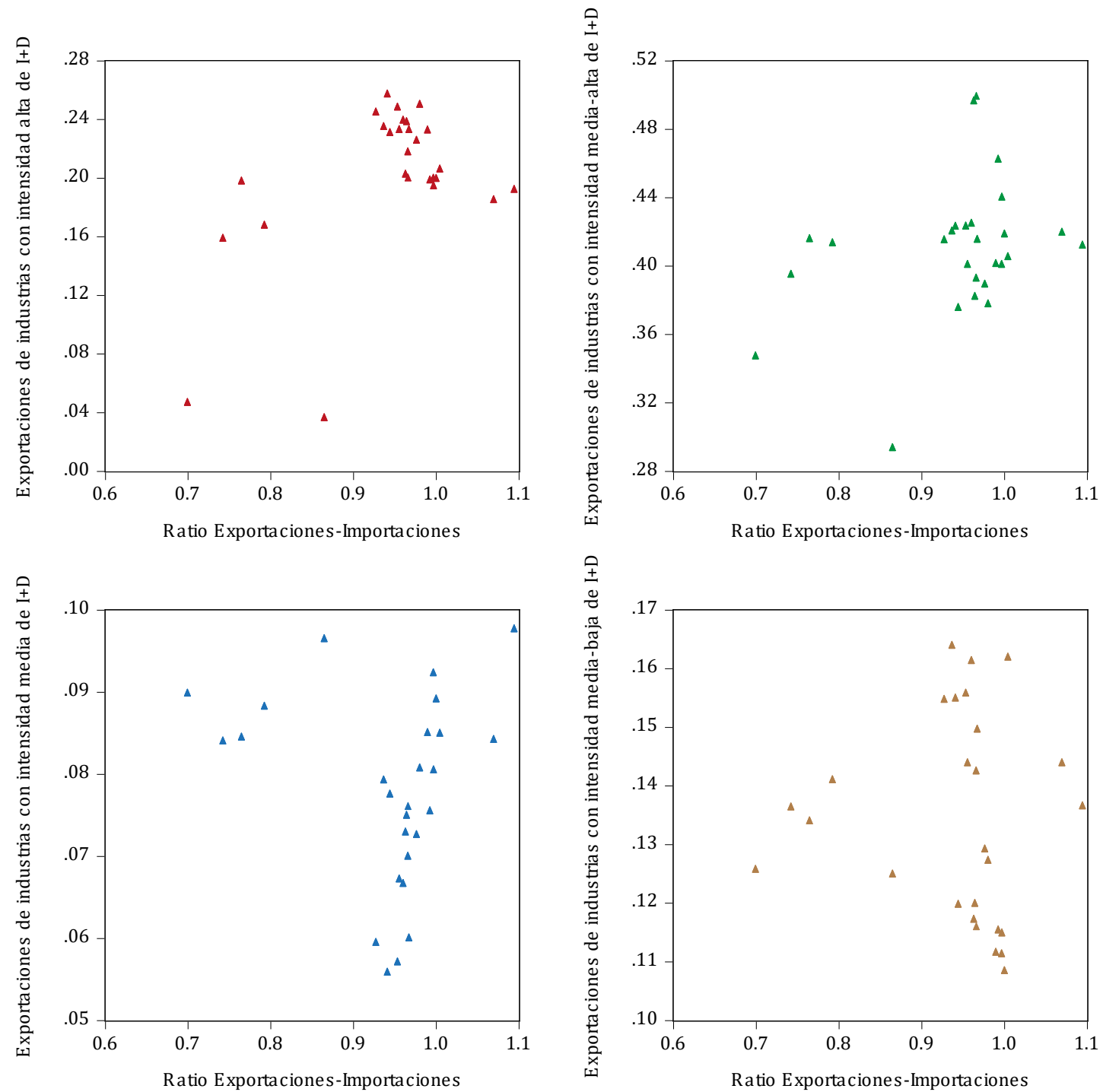

Fuente: elaboración propia a partir de la base de datos STAN de la OCDE, utilizando el programa Eviews.

cambien, pues, como se ha señalado, los factores que determinan el balance general pueden diferir, por su naturaleza, de los factores que determinan el balance bilateral (Khan y Hossain, 2012).

Así, existen indicios de la conexión entre las exportaciones de alta intensidad tecnológica y el balance bilateral de bienes. En términos del comercio bilateral de bienes, de la muestra inicial de 39 socios comerciales, México ha sido persistentemente superavitario con los Estados Unidos y Colombia, en donde la proporción de exportaciones de bienes de alta intensidad tecnológica hacia estos países es mayor al promedio. ¿Es esto una regularidad que estaría indicando la relación positiva entre exportaciones de industrias con intensidad tecnológica alta y el balance bilateral de bienes? La idea del ejercicio empírico que sigue es precisamente responder esta incógnita. 


\section{DATOS Y METODOLOGÍA}

Con datos anuales de 1990 a 2016, el análisis empírico se centra en el comercio bilateral entre México y una muestra de 39 socios comerciales ${ }^{2}$. La información del comercio bilateral fue extraída de la base de datos STAN Bilateral Trade Database (OCDE, 2017), que provee el valor de las exportaciones e importaciones bilaterales por sector industrial y diferentes categorías de uso. Los datos permiten identificar la composición de las exportaciones y las importaciones por intensidad tecnológica (intensidad alta de investigación y desarrollo [I+D], media-alta, media y media-baja). Por otra parte, los datos para estimar el tipo de cambio real bilateral y el ingreso relativo se obtuvieron de los Indicadores Mundiales de Desarrollo del Banco Mundial.

La construcción del modelo econométrico se sustenta en estudios empíricos previos donde el nivel de ingreso relativo y el tipo de cambio bilateral son las variables explicativas de mayor relevancia (Çelik y Kaya, 2010; Khan y Hossain, 2012; Gu, Zhou y Beg, 2014; Bineau, 2016). En la presente investigación el ingreso relativo se calcula como la razón entre el PIB per cápita del país socio $j$ y el PIB per cápita de México $\left(P I B_{j} / P I B_{M}\right)$, en dólares estadounidenses a precios constantes del 2010. El tipo de cambio real bilateral $\left(T C R_{M j}\right)$ se obtiene como el tipo de cambio nominal (pesos mexicanos por unidad de moneda extranjera) multiplicado por la razón entre el nivel de precios del socio $j$ y el nivel de precios en México ${ }^{3}$. Así, identificando a México con $M$ y a su socio comercial con $j$, la ecuación [1] presenta el modelo base:

2 Australia, Austria, Bélgica, Brasil, Canadá, China, Colombia, República Checa, Dinamarca, Estonia, Finlandia, Francia, Alemania, Grecia, Hungría, Islandia, India, Irlanda, Israel, Italia, Japón, Corea, Letonia, Luxemburgo, Holanda, Nueva Zelanda, Noruega, Polonia, Portugal, Rusia, Eslovaquia, Eslovenia, Sudáfrica, España, Suecia, Suiza, Turquía, Reino Unido y Estados Unidos

3 Para construir el tipo de cambio real bilateral de los países de la eurozona se obtuvo el tipo de cambio nominal a través de una triangulación entre el tipo de cambio de la moneda local utilizada antes de la adopción del euro y el dólar, y de la paridad fijada entre la moneda local y el euro en el momento de su entrada a la eurozona.
$\operatorname{Ln} B_{M j, t}=\beta_{0}+\beta_{1} \operatorname{Ln}\left(P I B_{j, t-1} / \mathrm{PIB}_{M, t-1}\right)$

$+\beta 2 \operatorname{LnTCR}_{M j, t-1}+e_{j}+v_{j t}$

Donde $B_{M, t}$ representa el balance bilateral entre México y su socio comercial $j$, medido como la razón entre exportaciones e importaciones de bienes. Un incremento relativo del nivel de ingreso del socio $j$ en relación con el de México $\left(P I B_{j} / P I B_{M}\right)$ impulsaría las exportaciones mexicanas, por lo que se esperaría una mejora del balance externo. Por su parte, un incremento en el tipo de cambio real bilateral $\left(T C R_{M j}\right)$ - una depreciación del peso mexicano-implicaría una mejora en la competitividad por precios de la economía mexicana, lo que se reflejaría en una mejora del balance externo. Las variables independientes son incluidas con un rezago para controlar posibles problemas de doble causalidad. Además, nótese que las variables son incluidas en logaritmos y, en consecuencia, los coeficientes miden elasticidades.

La presente investigación añade al modelo base la composición de las exportaciones $\left(C_{M, t}\right)$, medida como la razón entre las exportaciones de industrias con alta intensidad tecnológica y las exportaciones totales de bienes. La ecuación [2] representa el modelo extendido:

$$
\begin{aligned}
& \operatorname{Ln} B_{M j, t}=\beta_{0}+\beta_{1} \operatorname{Ln}\left(P I B_{j, t-1} / \mathrm{PIB}_{M, t-1}\right) \\
& +\beta 2 \operatorname{LnTCR}_{M j, t-1}+\beta_{3} \operatorname{Ln} C_{M, t,-1}+e_{j}+v_{j t}
\end{aligned}
$$

Para identificar la mejor estrategia de estimación, en primer lugar se implementaron pruebas de raíz unitaria que no requieren de un panel balanceado, esto es, la propuesta de Im, Pesaran, y Shin (2003), y las pruebas tipo Fisher (Maddala y Wu, 1999) utilizando ADF (Dickey y Fuller, 1979, 1981) y Phillips-Perron (1988). Los resultados de las pruebas de raíz unitaria (no reportados en tablas) señalan que únicamente la variable del ingreso relativo presenta raíz unitaria (estacionaria en primeras diferencias). Por consiguiente, el análisis de cointegración no es factible.

Debido al carácter autorregresivo de la balanza comercial, y a la posibilidad de algún grado de endogeneidad en las variables, se usó un modelo 
dinámico con datos de panel y un método general de momentos que en la literatura econométrica se conoce como método SYS GMM (Blundell y Bond, 1998). El método en mención incluye como variable explicativa el rezago de la variable dependiente, y utiliza rezagos en niveles y en primeras diferencias de la variable dependiente y de las independientes como instrumentos, mitigando así problemas de endogeneidad.

La estimación SYS GMM es eficiente y consistente si no hay presencia de autocorrelación, específicamente de segundo orden, lo que se verifica con la prueba de Arellano-Bond, y si los instrumentos son validados, lo que se verifica con la prueba de Sargan (Baltagi, 2005). En esta investigación se usó el estimador SYS GMM en dos etapas y se permitió un máximo de dos rezagos para ser incluidos como instrumentos, evitando así el problema de tener demasiados instrumentos (Roodman, 2009).

\section{RESULTADOS}

La tabla 1 presenta los principales resultados derivados del análisis de regresión. Es importante notar que el modelo dinámico está bien justificado, ya que la variable dependiente (balance comercial) rezagada e incluida como explicativa presenta significancia estadística. Además, las pruebas de autocorrelación de segundo orden y de validación de los instrumentos son superadas en todos los casos. Obsérvese que el estimador SYS GMM proporciona los coeficientes de corto plazo. Para conocer los coeficientes de largo plazo, hay que dividir el coeficiente de corto plazo entre uno menos el coeficiente de la variable dependiente como regresor.

La columna 1 de la tabla 1 muestra los coeficientes estimados para el modelo base. A favor de la hipótesis de trabajo, el tipo de cambio real bilateral evidencia un signo positivo y estadísticamente significativo; esto es, a una depreciación del peso corresponde una mejora en la balanza comercial bilateral, en línea con la condición Marshall-Lerner. En cambio, el ingreso relativo $\left(P I B_{j} / P I B_{M}\right)$ muestra un signo negativo y significancia estadística; es decir, un incremento en el PIB per cápita del socio comercial en relación con el PIB per cápita de México tiene un efecto negativo en la balanza comercial (una reducción en las exportaciones netas de bienes), lo cual contradice el efecto teórico esperado.

Para verificar la robustez de los resultados enunciados, se agregaron al modelo variables dicotómicas para los socios comerciales que forman parte de Norteamérica, América Latina y Europa Occidental (columna 2). Además, se incluyeron variables dicotómicas para los años de crisis económica, lo que permite controlar cambios coyunturales (columna 3).

Nótese que en diciembre de 1994 (año de inicio del TLCAN) y particularmente en 1995 México sufrió la llamada crisis del Tequila, donde la tasa de crecimiento fue de $-5,8 \%$. En 2001 la tasa de crecimiento del PIB también fue negativa, $-0,6 \%$, y finalmente en 2009 la tasa de crecimiento fue de $-4,7 \%$. No obstante, 2008 marcó el inicio de la última gran crisis financiera global y sus efectos siguieron siendo bastante fuertes en algunos países europeos hasta 2013. Además, las tasas negativas del PIB per cápita de México fueron mayores e incluyen más años: $1995(-7,5 \%), 2001(-1,9 \%)$, $2002(-1,1 \%), 2008(-0,2 \%), 2009(-6,2 \%)$ y $2013(-0,1 \%)$. Por lo tanto, se incluyen variables dicotómicas para estos años y 1994.

El tipo de cambio real confirma el efecto positivo sobre la balanza comercial, aun con la inclusión de las variables dicotómicas por región y años de crisis. En cambio, el efecto del ingreso relativo no es robusto. Aunque el coeficiente cambió a signo positivo, la inclusión de los años de crisis le hace perder significancia estadística.

Con respecto a las variables dicotómicas, en general se puede decir que los resultados sugieren un efecto negativo sobre la balanza comercial bilateral en el caso de los países europeos occidentales. En cambio, el efecto es positivo en el caso de los países latinoamericanos (en comparación con el grupo de referencia, formado principalmente por países asiáticos, de Europa Oriental y Oceanía). Además, los años de crisis tienen un impacto positivo sobre 
Tabla 1.

Principales resultados del análisis de regresión (SYS GMM en dos etapas)

\begin{tabular}{|c|c|c|c|c|c|}
\hline Variable/ítem & 1 & 2 & 3 & 4 & 5 \\
\hline Dependiente rezagada $\left(\mathrm{B}_{\mathrm{M}, \mathrm{t}-1}\right)$ & $0,36^{* * *}$ & $0,33^{* * *}$ & $0,35^{* * *}$ & $0,55^{* * *}$ & $0,53^{* * *}$ \\
\hline Ingreso relativo $\left(\mathrm{PIB}_{\mathrm{i}} / \mathrm{PIB}_{\mathrm{M}}\right)$ & $-0,20^{* * *}$ & 0,06 & 0,005 & $-0,28$ & \\
\hline Tipo de cambio real bilateral $\left(\mathrm{TCR}_{\mathrm{Mi}}\right)$ & $0,07^{* * *}$ & $0,09 * * *$ & $0,18^{* * *}$ & $0,20^{*}$ & \\
\hline Composición de las exportaciones $\left(C_{M, t}\right)$ & & & & $0,02^{* * *}$ & 0,09 \\
\hline Interacción $1\left(C_{M j, t}\right) \times\left(\mathrm{PIB}_{\mathrm{j}} / \mathrm{PIB}_{\mathrm{M}}\right)$ & & & & & $-0,30$ \\
\hline Interacción $2\left(C_{M j, t}\right) \times\left(T C R_{M j}\right)$ & & & & & $0,24^{* *}$ \\
\hline Norteamérica & & 0,44 & 0,22 & 2,69 & 2,67 \\
\hline América Latina & & $2,94^{* * *}$ & 0,71 & 1,35 & 0,69 \\
\hline Europa Occidental & & $-0,59 * * *$ & $-0,99 * * *$ & $-1,20^{* * *}$ & $-1,20^{* * *}$ \\
\hline Dummy 1994 & & & 0,07 & $0,36^{* * *}$ & $0,32^{* * *}$ \\
\hline Dummy 1995 & & & $0,60 * * *$ & $0,77^{* * *}$ & $0,77^{* * *}$ \\
\hline Dummy 2001 & & & $0,19^{* *}$ & $-0,06$ & $-0,07$ \\
\hline Dummy 2002 & & & $0,18^{* * *}$ & 0,06 & 0,06 \\
\hline Dummy 2008 & & & $-0,07$ & $-0,13^{* *}$ & $-0,15^{* *}$ \\
\hline Dummy 2009 & & & $-0,28^{* * *}$ & $-0,27^{* * *}$ & $-0,24^{* * *}$ \\
\hline Dummy 2013 & & & $-0,12^{* * *}$ & $-0,15^{* * *}$ & $-0,16^{* * *}$ \\
\hline Observaciones & 960 & 960 & 960 & 940 & 940 \\
\hline $\mathrm{N} \times \mathrm{T}$ & $39 \times 26$ & $39 \times 26$ & $39 \times 26$ & $39 \times 26$ & $39 \times 26$ \\
\hline Prueba de Sargan (significancia) & $\begin{array}{l}38,09 \\
(0,99) \\
\end{array}$ & $\begin{array}{l}36,36 \\
(0,99) \\
\end{array}$ & $\begin{array}{l}34,79 \\
(0,99) \\
\end{array}$ & $\begin{array}{l}30,56 \\
(1,00)\end{array}$ & $\begin{array}{l}31,06 \\
(1,00)\end{array}$ \\
\hline $\begin{array}{c}\text { Correlación serial de primer orden } \\
\text { (significancia) }\end{array}$ & $\begin{array}{c}-3,50 \\
(0,0005)\end{array}$ & $\begin{array}{c}-3,44 \\
(0,0006)\end{array}$ & $\begin{array}{c}-3,67 \\
(0,0002)\end{array}$ & $\begin{array}{l}-4,27 \\
(0,00)\end{array}$ & $\begin{array}{l}-4,20 \\
(0,00)\end{array}$ \\
\hline $\begin{array}{l}\text { Correlación serial de segundo orden } \\
\text { (significancia) }\end{array}$ & $\begin{array}{c}0,03 \\
(0,97)\end{array}$ & $\begin{array}{l}-0,08 \\
(0,93)\end{array}$ & $\begin{array}{c}0,60 \\
(0,54)\end{array}$ & $\begin{array}{c}1,04 \\
(0,29)\end{array}$ & $\begin{array}{c}1,05 \\
(0,29)\end{array}$ \\
\hline \multicolumn{6}{|c|}{ Coeficientes de largo plazo } \\
\hline Ingreso relativo (PIBj/PIBM) & $-0,31$ & 0,09 & 0,01 & $-0,62$ & \\
\hline Tipo de cambio real bilateral (TCRMj) & 0,11 & 0,13 & 0,28 & 0,44 & \\
\hline Composición de las exportaciones $\left(C_{M, t}\right)$ & & & & 0,04 & 0,19 \\
\hline
\end{tabular}

Nota: ${ }^{* *} \mathrm{y}^{* * *}$ indican respectivamente significancia estadística al $10 \%$, el $5 \%$ y el 1\%.

Fuente: elaboración propia con el programa Stata.

la balanza comercial — como es de esperar-, por cuanto las importaciones mexicanas tendrían que disminuir en dichos años. Sin embargo, los años de la última crisis financiera global indican que esta no solo ocurre en México, sino en todo el mundo, y particularmente en varios de los principales socios comerciales de México, que dejaron de comprarle reduciendo las exportaciones mexicanas. En particular, los coeficientes de los años 2009 y 2013 son negativos y significativos; por consiguiente, la crisis financiera global muestra un efecto negativo sobre la balanza comercial bilateral.

Continuando con la estimación del modelo extendido (ecuación [2]), se estima el modelo incluyendo la composición de las exportaciones y las variables dicotómicas (columna 4), y posteriormente se añaden términos de interacción (columna 5), excluyendo, por problemas de multicolinealidad, las variables sobre ingreso relativo y tipo de cambio.

Los resultados confirman los hallazgos previos y además permiten subrayar la relevancia de la composición de las exportaciones, cuyo coeficiente es positivo y estadísticamente significativo, lo cual implica que la balanza comercial bilateral de México tiende a mejorar conforme se incrementa la proporción de bienes de alta tecnología exportados. No obstante, este resultado debe considerarse como débil, porque la variable pierde significancia estadística al incluir los términos de interacción, donde solamente la interacción entre la composición 
de las exportaciones y el tipo de cambio presenta significancia estadística y un signo positivo. En varias otras especificaciones con y sin dummies para región y años de crisis, la composición de las exportaciones presenta signo positivo y significancia estadística, pero los términos de interacción sugieren que es un efecto que sucede gracias al impacto indirecto en el tipo de cambio.

Adicionalmente, se realizaron varias pruebas para verificar la robustez de los resultados previamente enunciados. En primer lugar, se estimaron los coeficientes del modelo base y sus extensiones utilizando otros métodos: el estimador DIF GMM (Arellano y Bond, 1991), el estimador con efectos fijos con la corrección propuesta por Bruno (2005) y modelos con efectos fijos y flexibles. Las propiedades del SYS GMM superan a la de estos otros métodos; en particular, las regresiones con efectos fijos y flexibles están sesgadas por problemas de endogeneidad. Algunos de estos resultados están reportados en la tabla 2 , donde no se encuentran resultados que contradigan lo obtenido del SYS GMM. Un resultado que conviene subrayar es la significancia estadística y el signo positivo de la

Tabla 2.

Principales resultados del análisis de regresión (DIF GMM, efectos fijos y flexibles)

\begin{tabular}{|c|c|c|c|c|c|}
\hline Variable/ítem & $\begin{array}{l}\text { DIF } \\
\text { GMM }\end{array}$ & DIF GMM & $\begin{array}{l}\text { Bruno } \\
(2005)\end{array}$ & Fijos & Flexibles \\
\hline Dependiente rezagada $\left(B_{M j, t-1}\right)$ & $0,46^{* * *}$ & $0,47^{* * *}$ & $0,51^{* * *}$ & $0,54^{* * *}$ & $0,76^{* * *}$ \\
\hline Ingreso relativo $\left(P I B_{j} / P I B_{M}\right)$ & $0,66^{*}$ & & 0,25 & 0,44 & $-0,01$ \\
\hline Tipo de cambio real bilateral $\left(T C R_{M j}\right)$ & $0,83^{* * *}$ & & $0,34^{* *}$ & $0,48^{* * *}$ & 0,02 \\
\hline Composición de las exportaciones $\left(C_{M j, t}\right)$ & $0,03^{* * *}$ & $-1,53^{* * *}$ & $-0,07^{* * *}$ & $-0,002$ & 0,02 \\
\hline Interacción $1\left(C_{M, t}\right) \times\left(P I B_{j} / P I B_{M}\right)$ & & $0,68^{*}$ & & & \\
\hline Interacción $2\left(C_{M j, t}\right) \times\left(T C R_{M j}\right)$ & & $0,89^{* * *}$ & & & \\
\hline Norteamérica & & & & & $0,37^{* * *}$ \\
\hline América Latina & & & & & $0,43^{* *}$ \\
\hline Europa Occidental & & & & & 0,03 \\
\hline Dummy 1994 & $0,68^{* * *}$ & $0,71^{* * *}$ & $-0,06$ & $0,31^{* *}$ & $0,39 * * *$ \\
\hline Dummy 1995 & $1,03^{* * *}$ & $1,07^{* * *}$ & $0,55^{* * *}$ & $0,83^{* * *}$ & $0,82^{* * *}$ \\
\hline Dummy 2001 & $0,10^{* * *}$ & $0,11^{* * *}$ & 0,04 & 0,02 & $-0,15$ \\
\hline Dummy 2002 & $0,22^{* * *}$ & $0,23^{* * *}$ & 0,04 & 0,16 & $-0,04$ \\
\hline Dummy 2008 & $0,06^{* *}$ & 0,04 & 0,07 & 0,09 & 0,15 \\
\hline Dummy 2009 & $-0,18^{* * *}$ & $-0,18^{* * *}$ & $-0,18$ & $-0,17$ & $-0,12$ \\
\hline Dummy 2013 & $-0,18^{* * *}$ & $-0,19 * * *$ & $-0,06$ & $-0,09$ & $-0,08$ \\
\hline Observaciones & 890 & 890 & 862 & 940 & 940 \\
\hline $\mathrm{N} \times \mathrm{T}$ & $39 \times 25$ & $39 \times 25$ & $39 \times 25$ & $39 \times 26$ & $39 \times 26$ \\
\hline R cuadrada & & & & 0,37 & 0,67 \\
\hline Prueba de Sargan (significancia) & $\begin{array}{l}31,99 \\
(0,96)\end{array}$ & $\begin{array}{l}32,82 \\
(0,95)\end{array}$ & & & \\
\hline $\begin{array}{l}\text { Correlación serial de primer orden } \\
\text { (significancia) }\end{array}$ & $\begin{array}{l}-4,23 \\
(0,00)\end{array}$ & $\begin{array}{l}-4,27 \\
(0,00)\end{array}$ & & & \\
\hline $\begin{array}{l}\text { Correlación serial de segundo orden } \\
\text { (significancia) }\end{array}$ & $\begin{array}{c}1,01 \\
(0,31)\end{array}$ & $\begin{array}{c}1,02 \\
(0,30)\end{array}$ & & & \\
\hline
\end{tabular}

Nota: ${ }^{* *} \mathrm{y}^{* * *}$ indican respectivamente significancia estadística al 10\%, el 5\% y el 1\%.

Fuente: elaboración propia con el programa Stata. 
composición de las exportaciones cuando se utiliza el estimador DIF GMM, así como su cambio a signo negativo con la inclusión de los términos de interacción. Lo anterior destaca la debilidad del efecto positivo sobre la balanza comercial. Igualmente, cabe resaltar que la variable dependiente como regresor es estadísticamente significativa en todos los casos, soportando la decisión de usar un modelo dinámico como el SYS GMM.

También se realizaron pruebas sin la transformación logarítmica, sin rezagos en las variables independientes, y reduciendo el tamaño de muestra a 20, 15 y 10 socios comerciales debido a las características del comercio internacional de México, altamente concentrado en unos pocos países, principalmente con los Estados Unidos. Los resultados (no reportados por espacio) son esencialmente parecidos a los ya enunciados.

\section{CONCLUSIONES E IMPLICACIONES}

Desde los ochenta, la persistencia del déficit en la cuenta corriente y en la balanza comercial de México ha despertado preocupación por su sostenibilidad y la restricción que impone en el crecimiento. En ese contexto, la presente investigación actualiza el efecto que tienen el nivel del ingreso y el tipo de cambio real sobre la balanza comercial, y añade a la explicación el papel de la composición de las exportaciones. Además, los datos y la estrategia empírica superan las limitaciones de las pruebas empíricas previas para México, basadas en series de tiempo y datos comerciales con los Estados Unidos. Los hallazgos de la presente investigación coinciden en cuanto a la importancia del tipo de cambio, pero discrepan en lo concerniente al impacto del nivel de ingreso. Aquí no se encontró evidencia robusta del nexo entre el ingreso relativo y la balanza comercial bilateral.

Trabajos previos sugieren una relación estable de largo plazo entre el nivel de ingreso (PIB o índices de producción industrial o actividad económica) y la balanza comercial (Galindo y Guerrero, 1997; Varela, 1999; Garcés, 2008; Baltazar y Ramos,
2014; Arriaga y Landa, 2016; Lagunes y Pérez, 2016; Márquez, 2018). Excluyendo el sector maquilador, Baltazar y Ramos (2014) señalan que en el caso de México hay elasticidad con respecto al ingreso e inelástico con respecto al tipo de cambio. Una excepción, además de lo aquí hallado, en cuanto al papel del nivel de ingreso, se puede encontrar en el trabajo de Márquez (2018), donde incluso se señala que hay una relación inversa entre la producción industrial de los Estados Unidos y la balanza comercial mexicana durante el periodo 2000-2014. Cabe señalar que el autor reconoce la inconsistencia con la teoría y que podría ser resultado de deficiencias del índice de producción estadounidense para aproximar al nivel de ingreso.

La principal diferencia entre este trabajo y los trabajos previos es que estos asumieron que el nivel de ingreso de los Estados Unidos equivalía al nivel de ingreso del resto del mundo, con lo cual sesgaban sus resultados. Aunque los citados trabajos también usan el tipo de cambio real con respecto al dólar estadounidense, con un sesgo similar al enunciado para el nivel de ingreso, los hallazgos del presente trabajo coinciden en que, en el largo plazo, la depreciación del peso mexicano - frente a cada una de las monedas de los 39 socios comerciales analizados - mejora la posición de su balanza comercial.

Ahora bien, la contribución más notable de la presente investigación consiste en reconocer que la composición de las exportaciones también tiene efectos sobre la balanza comercial. Los resultados coinciden con estudios previos que argumentan que los países que exportan productos con alto contenido tecnológico pueden mejorar el desempeño de sus exportaciones (Wierts, Van Kerkhoff y De Haan, 2014). No obstante, en el caso de México la evidencia del impacto de la composición de las exportaciones es débil; al incluir términos de interacción, la variable pierde significancia estadística, lo que puede relacionarse con aspectos de las exportaciones mexicanas que ya se han subrayado en la literatura: el sector maquilador en México es dominante, y eso afecta negativamente la cantidad de valor añadido que tienen las exportaciones (Arriaga y Landa, 2016). 
Adicionalmente, los efectos del incremento en las exportaciones sobre el crecimiento económico han sido limitados, lo que se debe, por un lado, a que los productos importados se apoderaron del mercado de bienes - particularmente los bienes intermedios - después del proceso de apertura comercial (Fujii, 2000) y, por otro, a los efectos limitados del sector exportador maquilador por las débiles conexiones con el resto de la economía y su bajo valor añadido (Fujii, Candaudap y Gaona, 2005).
Por tanto, una discusión impostergable invita a establecer las condiciones necesarias para que exista una relación positiva entre el sector exportador y el desempeño económico. Son tres los puntos por discutir: a) la necesidad de un mayor contenido de bienes intermedios en las exportaciones (Fujii y Cervantes, 2013); b) la implementación de una nueva política industrial (Wade, 2010; Calderón y Sánchez, 2012); y c) las fuentes de financiación y el alcance de la nueva política industrial.

\section{REFERENCIAS}

1. Aditya, A. y Acharyya, R. (2013). Export diversification, composition, and economic growth: Evidence from cross-country analysis. The Journal of International Trade Ë Economic Development, 22 (7), 959-992. https://doi.org/10.1080/09638199.2011.619009

2. Arellano, M. y Bond, S. (1991). Some tests of specification for panel data: Monte Carlo evidence and an application to employment equations. The Review of Economic Studies, 58(2), 277-297. https://doi. org/10.2307/2297968

3. Arriaga Navarrete, R. y Landa Díaz, H. O. (2016). Competitividad del sector externo mexicano: un análisis de la condición Marshall-Lerner. Revista Mexicana de Economía y Finanzas, 11(1), 79-101. https://www. remef.org.mx/index.php/remef/article/view/78

4. Bahmani-Oskooee, M. y Wang, Y. (2006). The J curve: China versus her trading partners. Bulletin of Economic Research, 58(4), 323-343. https://doi.org/10.1111/sjpe.12175

5. Bahmani-Oskooee, M., Hegerty, S. W. y Xu, J. (2013). Exchange-rate volatility and US-Hong Kong industry trade: is there evidence of a 'third country' effect? Applied Economics, 45(18), 2629-2651. http:// dx.doi.org/10.1111/twec. 12521

6. Bahmani-Oskooee, M. y Saha, S. (2017). Asymmetric response of the US-India trade balance to exchange rate changes: Evidence from 68 industries. The World Economy, 40(10), 2226-2254. https://doi. org/10.1111/j.0307-3378.2006.00247.x

7. Bahmani-Oskooee, M. y Harvey, H. (2018). The U.S. Trade balance with partners from developing world: An asymmetry analysis of the J-curve effect. Journal of Economic Development, 43(2), 29-43. https://pennstate. pure.elsevier.com/en/publications/the-us-trade-balance-with-partners-from-developing-world-an-asymm

8. Bahmani-Oskooee, M., Harvey, H. y Hegerty, S. W. (2018). The real peso-dollar rate and US-Mexico industry trade: An asymmetric analysis. Scottish Journal of Political Economy, 65(4), 350-389. https://doi. org/10.1080/00036846.2012.654918

9. Baltagi, B. (2005). Econometric analysis of panel data (3. ${ }^{\text {a }}$ ed.). Chichester: Johnv Wiley \& Sons.

10. Baltazar Escalona, J. C. y Ramos Escamilla, M. (2014). México: comercio exterior, producto y condición de Marshall-Lerner. Tiempo Económico, 27(9), 5-26. Https//doi.org/10.13140/RG.2.2.29436.54409

11. Bineau, Y. (2016). Real exchange rate and bilateral trade balance of Cambodia: A panel investigation. Economics Bulletin, 36(2), 895-900. https://ideas.repec.org/a/ebl/ecbull/eb-16-00075.html 
12. Blanchard, E. J. y Olney, W. W. (2017). Globalization and human capital investment: Export composition drives educational attainment. Journal of International Economics, 106, 165-183. https://doi.org/10.1016/j. jinteco.2017.03.004

13. Blundell, R. y Bond, S. (1998). Initial conditions and moment restrictions in dynamic panel data models. Journal of Econometrics, 87(1), 115-143. https://doi.org/10.1016/S0304-4076(98)00009-8

14. Botta, A. (2014). Structural asymmetries at the roots of the eurozone crisis: What's new for industrial policy in the EU? PSL Quarterly Review, 67(269), 169-216. http://dx.doi.org/10.2139/ssrn.2420168

15. Bravo-Ortega, C., Benavente, J. M. y González, Á. (2014). Innovation, exports, and productivity: Learning and self-selection in Chile. Emerging Markets Finance and Trade, 50(s1), 68-95. https://doi.org/10.2753/ REE1540-496X5001S105

16. Brissimis, S. N., Hondroyiannis, G., Papazoglou, C., Tsaveas, N. T. y Vasardani, M. A. (2013). The determinants of current account imbalances in the euro area: A panel estimation approach. Economic Change and Restructuring, 46(3), 299-319. https://doi.org/10.1007/s10644-012-9129-0

17. Bruno, G. S. F. (2005). Estimation and inference in dynamic unbalanced panel-data models with a small number of individuals. Stata Journal, 5(4), 473-500. https://EconPapers.repec.org/RePEc:tsj:stataj:v:5: y:2005:i:4:p:473-500

18. Calderon, C. A., Chong, A. y Loayza, N. V. (2002). Determinants of current account deficits in developing countries. Contributions in Macroeconomics, 2(1). https://doi.org/10.2202/1534-6005.1021

19. Calderón, C. y Sánchez, I. (2012). Crecimiento económico y política industrial en México. Problemas del Desarrollo, 170(4), 125-154. http://dx.doi.org/10.22201/iiec.20078951e.2012.170.32138

20. Carrasco, C. A. y Hernandez-del-Valle, A. (2017). Revisiting the factors behind European external imbalances. Journal of Economic Integration, 32 (2), 324-357. https://doi.org/10.11130/jei.2017.32.2.324

21. Carrasco, C. A. y Peinado, P. (2015). On the origin of european imbalances in the context of European integration. Panoeconomicus, 62(2), 177-191. https://doi.org/10.2298/PAN1502177C

22. Carrasco, C. A. y Serrano, F. (2015). Global and European imbalances and the crisis: A critical review. En The demise of finance-dominated capitalism (pp. 265-288). Cheltenham: Edward Elgar Publishing.

23. Çelik, S. y Kaya, H. (2010). Real exchange rates and bilateral trade dynamics of Turkey: Panel cointegration approach. Applied Economics Letters, 17(8), 791-795. https://doi.org/10.1080/13504850802388993

24. Cooper, J. C. B. (2003). Price elasticity of demand for crude oil: estimates for 23 countries. OPEC Review, 27(1), 1-8. https://doi.org/10.1111/1468-0076.00121

25. Dickey, D. A. y Fuller, W. A. (1979). Distribution of the estimators for autoregressive time series with a unit root. Journal of the American Statistical Association, 74(366), 427-431. https://doi.org/10.2307/2286348

26. Dickey, D. A. y Fuller, W. A. (1981). Likelihood ratio statistics for autoregressive time series with a unit root. Econometrica, 49(4), 1057-1072. https://doi.org/10.2307/1912517

27. Dooley, M. P. (2000). A model of crises in emerging markets. The Economic Journal, 110(460), 256-272. https://doi.org/10.1111/1468-0297.00500 
28. Fosu, A. K. (1990). Export composition and the impact of exports on economic growth of developing economies. Economics Letters, 34(1), 67-71. https://doi.org/10.1016/0165-1765(90)90183-2

29. Fujii, G. (2000). El comercio exterior manufacturero y los límites al crecimiento económico de México. Comercio Exterior. http://revistas.bancomext.gob.mx/rce/magazines/42/9/RCE.pdf

30. Fujii, G., Candaudap, E. y Gaona, C. (2005). Exportaciones, industria maquiladora y crecimiento económico en México a partir de la década de los noventa. Investigación Económica, 64(254), 125-156. http:// www.scielo.org.mx/pdf/ineco/v64n254/0185-1667-ineco-64-254-125.pdf

31. Fujii, G. y Cervantes, R. (2013). México: valor agregado en las exportaciones manufactureras. Revista CEPAL, 109, 143-158. https://repositorio.cepal.org/handle/11362/11575

32. Galindo, L. M. y Guerrero, C. (1997). Factores determinantes de la balanza comercial de México 19801995. Comercio Exterior, 10, 789-794. http://revistas.bancomext.gob.mx/rce/magazines/340/2/RCE2.pdf

33. Garcés Díaz, D. G. (2008). Análisis de las funciones de importación y exportación de México (19802000). El Trimestre Económico, 75(297), 109-141. http://www.eltrimestreeconomico.com.mx/index.php/ te/article/view/393/592

34. Ghatak, S., Milner, C. y Utkulu, U. (1997). Exports, export composition and growth: Cointegration and causality evidence for Malaysia. Applied Economics, 29(2), 213-223. https://doi.org/10.1080/000368497327272

35. Gu, X., Zhou, Z.-Y. y Beg, A. B. (2014). What determines China's trade balance dynamics: A disaggregate analysis of panel data. Journal of the Asia Pacific Economy, 19(2), 353-368. https://doi.org/10.1080/1354 7860.2014 .880284

36. Herzer, D., Nowak-Lehmann, F. y Siliverstovs, B. (2006). Export-led growth in Chile: Assessing the role of export composition in productivity growth. The Developing Economies, 44(3), 306-328. https://doi. org/10.1111/j.1746-1049.2006.00019.x

37. Im, K. S., Pesaran, M. H. y Shin, Y. (2003). Testing for unit roots in heterogeneous panels. Journal of Econometrics, 115(1), 53-74. https://doi.org/10.1016/S0304-4076(03)00092-7

38. Johnson, R. C. (2014). Five facts about value-added exports and implications for macroeconomics and trade research. Journal of Economic Perspectives, 28(2), 119-142. https://doi.org/10.1257/jep.28.2.119

39. Khan, M. Z. S. y Hossain, M. I. (2010). A model of bilateral trade balance: Extensions and empirical tests. Economic Analysis and Policy, 40(3), 377-391. https://doi.org/10.1016/S0313-5926(10)50037-7

40. Khan, M. Z. S. y Hossain, M. I. (2012). Determinants of trade balance of Bangladesh: A dynamic panel data analysis. Bangladesh Development Studies, 35(2), 45-65. https://bids.org.bd/uploads/publication/ BDS/35/35-2/02_Determinants\%20of\%20Trade.pdf

41. Krichene, N. (2002). World crude oil and natural gas: A demand and supply model. Energy Economics, 24(6), 557-576. https://doi.org/10.1016/S0140-9883(02)00061-0

42. Krugman, P. R. y Baldwin, R. E. (1987). The Persistence of the U.S. Trade Deficit. Brookings Papers on Economic Activity, 1987(1), 1-55. https://core.ac.uk/download/pdf/6252194.pdf 
43. Lagunes Pérez, M. A. y Pérez Villarreal, H. H. (2016). Tipo de cambio y determinantes de las exportaciones en periodos de volatilidad financiera en la zona del tratado de libre comercio de Norteamérica. Revista Internacional Administración Eु Finanzas, 9(2), 61-71. ftp://ftp.repec.org/opt/ReDIF/RePEc/ibf/riafin/riafv9n2-2016/RIAF-V9N2-2016-5.pdf

44. Maddala, G. S. y Wu, S. (1999). A comparative study of unit root tests with panel data and a new simple test. Oxford Bulletin of Economics and Statistics, 61 (s1), 631-652. https://doi.org/10.1111/1468-0084.0610s1631

45. Márquez Ortiz, J. C. (2018). Determinantes de la balanza comercial en México 2000-2014. Managment Review, 3(1). https://doi.org/10.18583/umr.v3i1.104.g219

46. Mazzucato, M. et al. (2015). Which industrial policy does Europe need? Intereconomics, 50(3), 120-155. https://doi.org/10.1007/s10272-015-0535-1

47. Mishkin, F. (1996). Understanding financial crises: A developing country perspective. Cambridge: National Bureau of Economic Research.

48. Moreno-Brid, J. C. y Ros-Bosch, J. (2010). Desarrollo y crecimiento en la economía mexicana. Una perspectiva histórica. Ciudad de México: Fondo de Cultura Económica.

49. Organización para la Cooperación y el Desarrollo Económicos (OCDE) (2017). STAN Bilateral Database ISIC4. Recuperado de https://stats.oecd.org/Index.aspx?DataSetCode=BTDIXE

50. Phillips, P. C. B. y Perron, P. (1988). Testing for a unit root in time series regression. Biometrika, 75(2), 335. https://doi.org/10.2307/2336182

51. Roodman, D. (2009). A note on the theme of too many instruments. Oxford Bulletin of Economics and Statistics, $71(1), 135-158$. https://doi.org/10.1111/j.1468-0084.2008.00542.x

52. Secretaría de Economía (2015). Comercio exterior / Países con tratados y acuerdos firmados con México. Recuperado de https://www.gob.mx/se/acciones-y-programas/comercio-exterior-paises-con-tratados-y -acuerdos-firmados-con-mexico

53. Sheridan, B. J. (2014). Manufacturing exports and growth: When is a developing country ready to transition from primary exports to manufacturing exports? Journal of Macroeconomics, 42, 1-13. https://doi. org/10.1016/j.jmacro.2014.06.002

54. Srholec, M. (2007). High-tech exports from developing countries: A symptom of technology spurts or statistical illusion? Review of World Economics, 143(2), 227-255. https://doi.org/10.1007/s10290-007-0106-z

55. Tovar-García, E. D. (2018). Does the share of crude petroleum and natural gas in exports increase total exports? The Russian case. World Economy and International Relations, 62 (6), 30-35. https://doi. org/10.20542/0131-2227-2018-62-6-30-35

56. Tovar-García, E. D. y Carrasco, C. A. (2019). Export and import composition as determinants of bilateral trade in goods: Evidence from Russia. Post-Communist Economies, 1-17. https://doi.org/10.1080/146313 77.2018 .1557913

57. Varela Llamas, R. (1999). Factores determinantes del saldo de la balanza comercial en México, 1989-1998. Comercio Exterior, 10, 939-943. 
58. Wade, R. (2010). After the Crisis: Industrial Policy and the Developmental State in Low-Income Countries. Global Policy, 1(2), 150-161. https://doi.org/10.1111/j.1758-5899.2010.00036.x

59. Wierts, P., Van Kerkhoff, H. y De Haan, J. (2014). Composition of exports and export performance of Eurozone countries. Journal of Common Market Studies, 52 (4), 928-941. https://doi.org/10.1111/jcms.12114

60. Zakir, M. e Ismail, M. (2010). A model of bilateral trade balance: Extensions and empirical tests. Economic Analysis and Policy, 40(3), 377-391. https://doi.org/10.1016/S0313-5926(10)50037-7 